\title{
Soluble glucocorticoid-induced tumor necrosis factor receptor regulates Helios expression in myasthenia gravis
}

\author{
Yi Li ${ }^{1}$, Shumei Yang ${ }^{1}$, Zhibin Li ${ }^{1}$, Huanyu Meng ${ }^{1}$, Wanling Jin ${ }^{1}$, Huan Yang ${ }^{1 *}$ (D) and Weifan Yin² ${ }^{2^{*}}$
}

\begin{abstract}
Background: Helios is important for functional and phenotype stability of regulatory T cells (Tregs). However, the role of Helios in autoimmune diseases and its regulation remains unclear. This study aimed to investigate the role of Helios $^{+}$Tregs in myasthenia gravis (MG) and glucocorticoid-induced tumor necrosis factor receptor (GITR) and its ligand (GITRL) in the modulation of Helios.

Method: Multicolor flow cytometry was performed to analyze Helios ${ }^{+}$Tregs in peripheral blood from MG patients and healthy donors (HDs). Enzyme-linked immunosorbent assay (ELISA) was used to determine the levels of soluble GITRL/GITR in plasma. Tregs were isolated via magnetic separation and treated with recombinant GITRL and GITR-FC. Membrane GITRL on Tregs and expression of Helios and other markers (FOXP3, CD25, CD39, CTLA-4, PD-L1 and IL-10) involved in immunosuppressive activity were determined by flow cytometry.

Result: Both Helios ${ }^{+}$Tregs and soluble GITR were decreased in generalized MG (GMG) patients $(n=14)$, compared with HDs $(n=14)$ and ocular MG $(O M G)$ patients $(n=16)$. Helios ${ }^{+}$Tregs possessed greater immunosuppressive capacity compared to Helios ${ }^{-}$Tregs. Further analysis indicates soluble GITR was negatively correlated with quantitative MG score and promoted Helios expression and enhanced function of Tregs independently of membrane GITRL.

Conclusion: This work demonstrates abnormal changes in Helios ${ }^{+}$Tregs and soluble GITR in MG, as well as direct regulation of Helios by GITR in the context of Tregs. This work provides new insight into the role of GITR in the regulatory pathway of Helios and pathogenesis of MG.
\end{abstract}

Keywords: GITRL, GITR, Helios, Myasthenia gravis, Regulatory T cells

\section{Background}

Myasthenia gravis (MG) is T cell-dependent autoimmune disorder caused by antibodies targeting the neuromuscular junction, and affects $40-180$ per million people with an annual incidence of 4-12 per million people [1]. It has been widely accepted that imbalance between $\mathrm{T}$ helper cells and regulatory $\mathrm{T}$ cells (Tregs) plays an essential role in the pathogenesis of MG. Abnormalities in the quantity or function of Tregs have been demonstrated in multiple

\footnotetext{
*Correspondence: yangh69@126.com; yinweifan1985@csu.edu.cn

†Yang Huan and Yin Weifan contributed equally to this work

${ }^{1}$ Department of Neurology, Xiangya Hospital, Central South University, 87 Xiangya Road, Changsha, Hunan, China

2 Department of Neurology, Second Xiangya Hospital, Central South University, 137 People Road, Changsha, Hunan, China
}

studies of MG [2-4]. Investigating the key factors that modulate the function or phenotypic stability of Tregs is important for understanding the mechanism of MG and developing new therapeutic strategies.

Helios, a member of the Ikaros family of zinc finger proteins, was once thought to be a marker of thymic Tregs that were differentiated from peripheral Tregs $[5$, 6]. However, recent studies have suggested that Helios is involved in Treg function and phenotype stability. Helios expression in Tregs ensures a suppressive and anergic phenotype in intense inflammatory responses [7]. Overexpression of Helios enhances Treg function in cooperation with FOXP3 [7]. Selective Helios deficiency within Tregs led to induction of an unstable phenotype and conversion of Tregs into $\mathrm{T}$ effector cells within the tumor 
microenvironment and was associated with increased production of proinflammatory cytokines [8]. Helios deficiency in Tregs also led to reduced expansion of pathogenic $\mathrm{T}$ cells, $\mathrm{T}$ follicular helper cell and Th1 effector cell responses, and $\mathrm{T}$ follicular regulatory cell function [9]. However, the role of Helios ${ }^{+}$Tregs in autoimmune diseases is still unclear, and little is known about its regulatory pathway.

The glucocorticoid-induced tumor necrosis factor receptor (GITR, TNFRSF18) and its ligand glucocorticoid-induced tumor necrosis factor receptor ligand (GITRL, TNFSF18) are members of the tumor necrosis factor/tumor necrosis factor receptor (TNF/TNFR) superfamily. GITR is expressed on various types of immune cells and constitutively expressed at a high level in Tregs [9]. GITRL is found on antigen-presenting cells, including dendritic cells, macrophages [10, 11]. GITRL/ GITR has been reported to participate in the development of immune response against tumors and infectious agents, as well as in autoimmune and inflammatory diseases [12]. The role of GITRL in modulating $\mathrm{T}$ cell response is unclear, but most studies support that GITRL promotes the immune response and enhances tumor rejection [11, 13, 14]; however, GITR exerts anti-inflammatory effects and decreases the autoimmune response [12]. The underlying mechanisms of these responses remain unclear. A previous study suggested that triggering GITR with its antibody correlated with a drastic loss of Helios [15]. Thus, we suggest that GITRL/GITR may exert their function partially through the regulation of Helios in Tregs.

Therefore, we investigated the profile of Helios expressed on Tregs in MG and the immunosuppressive potential of Helios ${ }^{+}$Tregs versus Helios- Tregs. Further, we demonstrated that soluble GITR and GITRL (sGITRL/sGITR) facilitate Helios-independent membrane GITRL.

\section{Methods and materials Patients and samples}

Patients with possible MG consulting at the Neurology Department of Xiangya Hospital, Changsha, China, and healthy donors (HDs) were recruited from June 2017 to April 2018. Diagnostic criteria were based on fluctuating muscle weakness, positive neostigmine test, and abnormal repetitive nerve stimulation test (RNS). The enrolled subjects were screened for not having undergone any prior immune suppression therapy or thymectomy, and were also determined to be not suffering from any other autoimmune diseases or infection.

Clinical assessments were performed by neurologists in the MG Diagnosis and Treatment Center, Xiangya Hospital. Patients were instructed to not take any acetylcholine esterase inhibitor within $12 \mathrm{~h}$ before examination. During the examination, $5 \mathrm{~mL}$ blood samples were collected. Ocular MG (OMG) or generalized MG (GMG) was determined according to the Myasthenia Gravis Foundation of America (MGFA) clinical classification. The quantitative MG (QMG) score was performed when the patients were enrolled and treated by glucocorticoid 8-12 weeks (oral prednisolone, beginning with $20 \mathrm{mg} /$ day and gradually increasing to the target dose of $1 \mathrm{mg} /$ $\mathrm{kg} /$ day).

Patients with crisis or impending crisis treated with lymphoplasmapheresis were also selected, and their exchanged blood was used to isolate regulatory $\mathrm{T}$ cells. Isolated regulatory $\mathrm{T}$ cells were cultured for further drug treatment and flow cytometry. The levels of nicotinic acetylcholine receptor antibody (AchR-Ab) or muscle-specific tyrosine kinase antibody (MuSK-Ab) were acquired from the DAAN Clinical Laboratory Central Company (Guangzhou, China). Level out-weight of $0.45 \mathrm{nMol}$ was considered to be positive for AchR-Ab and $0.05 \mathrm{nMol}$ for MuSK-Ab.

All patients and HDs signed informed consent forms. The study was approved by the Ethics Review Committee of Xiangya Hospital.

\section{Enzyme-linked immunosorbent assay (ELISA)}

sGITR and sGITRL levels in plasma were determined by ELISA according to manufacturer's instructions. Levels of sGITRL were measured via a pre-coated GITRL Human ELISA Kit (Invitrogen, Carlsbad, CA, USA). Levels of sGITR were measured via a Sandwich ELISA Kit (RayBiotech Inc, Norcross, GA, USA).

\section{Flow cytometry}

Flow cytometry was performed on a FACS-Canto II system (BD Biosciences, San Jose, CA, USA). Blood mononuclear cells (PBMCs) or isolated Tregs were analyzed for surface expression or intracellular markers using the following anti-human antibodies: PE-Cy7-labeled antiCD4 (clone:SK3, BD Biosciences), Percp-Cy5.5-labeled anti-CD25 (clone: M-A251, BD Biosciences), APC-Cy7labeled anti-CD39 (clone: A1, BD Biosciences), BV421labeled anti-CTLA4 (clone: BNI3, BioLegend, San Diego, USA), PE-labeled anti-PD-L1 (clone: 29E2A3, BioLegend), Alexa Fluor 647-labeled anti-FOXP3 (clone: 259D/ C7, BD Biosciences), Alexa Fluor 488-labeled anti-Helios (clone: 22F6, BD Biosciences), PE-labeled IL-10(clone: JES3-19F1, BioLegend) and PE-labeled anti-GITRL(clone: REA841, Miltenyi Biotec, Bergisch Gladbach, Germany). For surface marker staining, cells were incubated with the antibodies for $30 \mathrm{~min}$ at $4{ }^{\circ} \mathrm{C}$ shielded from light, then washed with PBS. For intracellular transcription factor and IL-10 staining, cells were first permeabilized using 
the Cytofix/Cytoperm Fixation/Permeabilization Kit (BD Biosciences), and then cells were incubated with intracellular transfactor antibody in eBiosciences Staining Buffer (Thermo Fisher Scientific, Waltham, MA, USA) for $45 \mathrm{~min}$ at $4{ }^{\circ} \mathrm{C}$ shielded from light. For IL-10 staining, cells were stimulated by $5 \mu \mathrm{g} / \mathrm{ml}$ anti-CD3 monoclonal antibodies (clone: UCHT1, Merck-Millipore, Burlington, MA, USA) and $1 \mu \mathrm{g} / \mathrm{ml}$ anti-CD28 monoclonal antibodies (clone: 28.2, Biolegend) for $48 \mathrm{~h}$ and Brefeldin A Solution $(5 \mu \mathrm{g} / \mathrm{ml})$ was added at final $6 \mathrm{~h}$ before harvest. After staining, cells were washed and fixed with $1 \%$ paraformaldehyde. The LIVE/DEAD Fixable Dead Cell Stain Kit (Invitrogen) was used for exclusion of dead cells. The positivity for all markers was established based on fluorescence minus one (FMO) and isotypic controls. All data were analyzed by FlowJo software VX.

\section{PBMC and regulatory $\mathrm{T}$ cell isolation}

The peripheral blood samples collected in tubes with EDTA were centrifuged to obtain supernatant plasma, then diluted 1:1 with sterile PBS at room temperature $\left(18-25{ }^{\circ} \mathrm{C}\right)$ and centrifuged on lymphocyte isolation agent (TBD, Tianjin, China) at $900 \mathrm{~g}$ for $30 \mathrm{~min}$. The isolated PBMCs were collected and washed with PBS. Tregs were isolated from the PBMCs of the patients who had accepted lymphoplasmapheresis therapy using magnetic separation (Miltenyi Biotec), according to the manufacturer's instructions.

\section{Cell culture and drug treatment}

Isolated Tregs were seeded in a 96-well round-bottom plate at $2 \times 10^{5}$ cells per pore. Plates were coated overnight $(12-16 \mathrm{~h}$ ) with $10 \mu \mathrm{g} / \mathrm{mL}$ anti-CD3 mAb (clone: UCHT1, Merck-Millipore) before adding the cells. Cells were cultured in RPMI 1640 supplemented with Gibco $10 \%$ heat inactivated fetal bovine serum (Thermo Fisher Scientific), 1\% sodium pyruvate (Thermo Fisher Scientific), $10,000 \mathrm{U} / \mathrm{mL}$ penicillin, $100 \mathrm{mg} / \mathrm{mL}$ streptomycin and $2 \mathrm{mM}$ L-glutamine(Thermo Fisher Scientific). To investigate the effect of sGITRL/sGITR, Tregs were incubated with $0.2 \mu \mathrm{g} / \mathrm{mL}, 1 \mu \mathrm{g} / \mathrm{mL}$, or $5 \mu \mathrm{g} / \mathrm{mL}$ GITRL (Novus Biologicals, Centennial, CO, USA) or $0.4 \mu \mathrm{g} / \mathrm{mL}$, $2 \mu \mathrm{g} / \mathrm{mL}$, or $10 \mu \mathrm{g} / \mathrm{mL}$ GITR-Fc refusion protein (BioLegend) for $72 \mathrm{~h}$. Heat-inactivated GITRL $(5 \mu \mathrm{g} / \mathrm{mL})$ or human IgG (10 $\mu \mathrm{g} / \mathrm{mL}$, Dingguo, Beijing, China) were used as controls. Then, $10^{5}$ cells were collected and analyzed by flow cytometry.

\section{Statistical analysis}

Data are expressed as mean \pm SEM or median. The distribution of each parameter was evaluated by the Kolmogorov-Smirnov test. For data with normal distribution and homogeneity of variance, unpaired or paired
Student's $t$-test or one-way analysis of variance (ANOVA) was used. Tukey test was used for multiple comparison between different groups and post linear trend test was used to analyze the tendency. The Wilcoxon signed-rank test or Kruskal-Wallis test was used to compare data with a non-normal distribution, and Dunn's test was used for multiple comparison. Correlations were assessed by Spearman's rank correlation coefficients. A p-value $<0.05$ was considered significant. All statistical analyses were performed using GraphPad Prism software (version 5.0).

\section{Results}

Frequencies of Helios ${ }^{+}$Tregs in GMG patients decreased and were correlated to QMG scores

A total of 55 patients suspected to have MG were reviewed, and 41 were confirmed to be MG cases. Among these cases, 6 were concomitant autoimmune thyroiditis, 1 was concomitant pemphigus; 2 patients were infected with hepatitis B virus, 2 patients were suffering with tuberculosis, and 11 subjects were excluded. In total, 30 MG patients and 14 HDs were enrolled. Patient information is provided in Table 1 . There was no difference in age or sex ratio among each group. First, we analyzed the frequencies of Helios ${ }^{+}$Treg in peripheral blood from the OMG and GMG patients and HDs by flow cytometry. We gated in $\mathrm{CD}^{+}{ }^{+} \mathrm{CD} 25^{+} \mathrm{FOXP3}^{+}$and $\mathrm{Helios}^{+}$to distinguish Helios ${ }^{+}$Tregs in PBMCs (Fig. 1a, b). It was found that frequencies of Helios ${ }^{+}$Tregs in GMG patients were significantly lower than those in HDs (median values

\begin{tabular}{|c|c|c|c|c|}
\hline Groups & OMG & GMG & HD & $\mathrm{p}$ \\
\hline Number of cases & 16 & 14 & 14 & \\
\hline Age (mean $\pm S D)$ & $36.2 \pm 16.7$ & $44.29 \pm 9.21$ & $39.14 \pm 10.9$ & 0.238 \\
\hline Sex (male/female) & $9 / 7$ & $6 / 8$ & $6 / 8$ & 0.6935 \\
\hline QMG score (mean \pm SD) & $5.36 \pm 1.84$ & $16.14 \pm 7.37$ & & \\
\hline MGFA I & 16 & 0 & & \\
\hline MGFA ॥ & 0 & 5 & & \\
\hline MGFA III & 0 & 6 & & \\
\hline MGFA IV & 0 & 2 & & \\
\hline MGFA V & 0 & 1 & & \\
\hline AchR-Ab positive & 14 & 13 & & \\
\hline MuSK-Ab positive & 0 & 1 & & \\
\hline Seronegtive & 2 & 0 & & \\
\hline Thymoma & 0 & 1 & & \\
\hline
\end{tabular}

No significant differences were observed in age and gender ratio among three groups $(p>0.05)$

OMG ocular myasthenia gravis, GMG generalized myasthenia gravis, $H D$ healthy donors, MGFA Myasthenia Gravis Foundation of America Clinical Classification, $A c h R-A b$ nicotinic acetylcholine receptor antibody, MUSK-Ab muscle specific tyrosine kinase antibody 


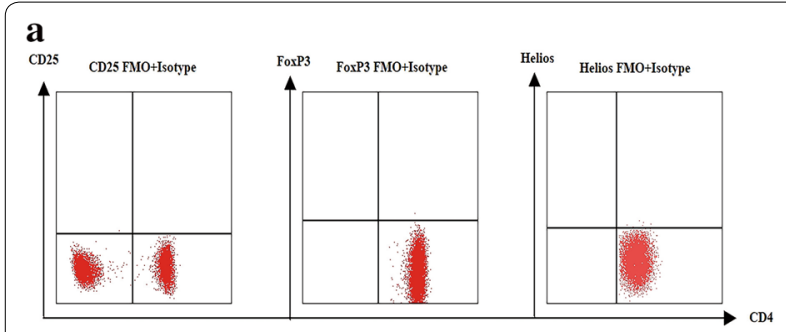

b

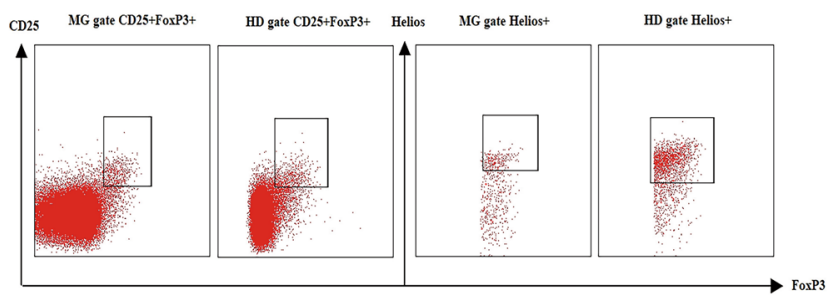

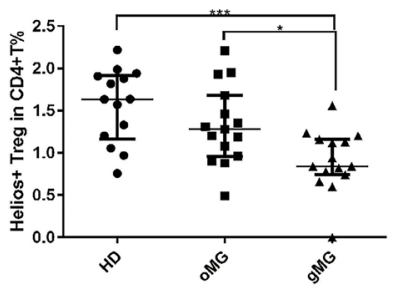

d

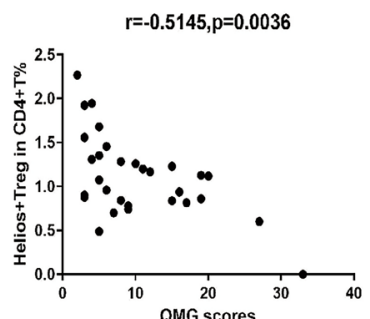

$\mathbf{e}$

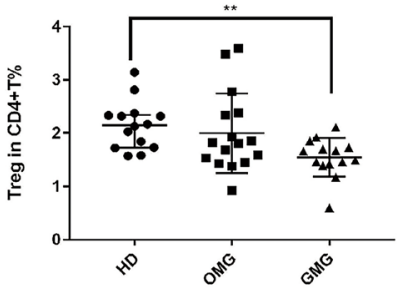

f

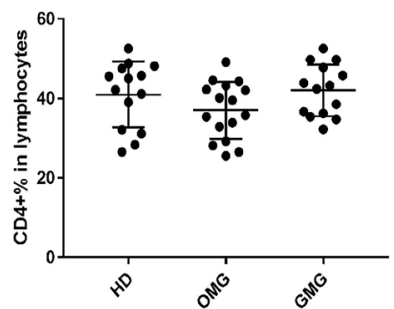

Fig. 1 Frequencies of Helios ${ }^{+}$Tregs in MG patients compared to HDs. a The FMO and isotype controls are shown. b Analysis of CD25, FOXP3, and Helios expression among $\mathrm{CD}^{+} \mathrm{T}$ cells in representative peripheral blood samples from HDs and patients with MG. $\mathbf{c}$ Frequencies of Helios ${ }^{+}$ Tregs among $\mathrm{CD}^{+}$T cells in HDs $(n=14)$, and patients with OMG $(n=14)$, and GMG $(n=16)$ (median/interquartile range values). $\mathbf{d}$ Correlation between frequencies of Helios ${ }^{+}$Tregs among CD4 ${ }^{+} T$ cells in MG patients with their QMG scores. e, $\mathbf{f}$ Frequency of Tregs in GMG patients decreased compared to $\mathrm{HDs}(p=0.0059)$ and there was no different of $\mathrm{CD}^{+}{ }^{+}$T frequency in each groups $(p=0.1435)\left({ }^{*} p<0.05,{ }^{* *} p<0.01,{ }^{* * *} p<0.005\right.$, $\left.{ }^{* * * *} p<0.001\right)$

$0.84 \%$ vs. $1.63 \%, p=0.0009$ ) and OMG patients (median values $0.84 \%$ vs. $1.28 \%, p=0.0402$ ), while there was no difference between OMG patients and HDs (median values $1.28 \%$ vs. $1.63 \%, p=0.6914)$. We also found that the frequency of Helios ${ }^{+}$Tregs were negatively correlated with QMG score in MG patients $(\mathrm{r}=-0.5145, p=0.0036$, Fig. 1c, d). The frequency of Tregs in GMG patients were also found decreased compared to HDs $(p=0.0059$, Fig. 1e) and there was no different of $\mathrm{CD}^{+} \mathrm{T}$ frequency in each groups $(p=0.1435$, Fig. 1f). No correlation was found between QMG scores and frequency of Tregs $(p=0.1646$, not shown).

\section{Glucocorticoid treatment increased frequency of Helios ${ }^{+}$ Tregs in MG patients}

In order to analyze if changes in $\mathrm{Helios}^{+}$Tregs were linked to treatment response, we performed longitudinal analysis of QMG and frequency of Helios ${ }^{+}$Tregs. In $10 \mathrm{MG}$ patients who received 8-12 weeks oral prednisolone therapy, the QMG scores were dramatically decreased ( $p=0.0039)$; accompanying with the frequency of Helios $^{+}$Tregs increased $(\mathrm{p}=0.0078)$ (Fig. 2).

\section{Helios $^{+}$Tregs expressed higher level of CD39 and FOXP3}

We next compared the expression of suppressive function-related molecules between $\mathrm{Helios}^{+}$Tregs and Helios $^{-}$Tregs. An additional $10 \mathrm{MG}$ patients and 10
HDs were recruited (Table 2). Blood samples were collected and analyzed by flow cytometry. We first gated $\mathrm{CD}^{+} \mathrm{CD}^{2} 5^{+} \mathrm{FOXP}^{+}$to distinguish Tregs. Then FOXP3, CD25, CD39, CTLA-4, PD-L1 and IL-10 were compared, respectively in $\mathrm{Helios}^{+}$subgroup and $\mathrm{Helios}^{-}$subgroup in these identified Tregs. The analysis shows that expression levels of FOXP3 and CD39 were higher in Helios ${ }^{+}$ Tregs than those in Helios ${ }^{-}$Tregs. On the other hand, the levels of the other markers related to immunosuppressive activity, such as CD25, CTLA-4, PD-L1 and IL-10 were comparable between Helios ${ }^{+}$Tregs and Helios ${ }^{-}$ Tregs, as shown in Fig. 3. Taken together, these results suggest that Helios ${ }^{+}$Tregs may be more suppressive than Helios $^{-}$Tregs.

\section{sGITR were decreased in GMG patients and correlated to frequency of Helios ${ }^{+}$Tregs}

Previous studies have revealed that Treg-mediated suppression of the immune response is abrogated by triggering GITR $[13,16]$ and high levels of sGITRL or SGITR in serum have been reported in autoimmune diseases, such as rheumatoid arthritis (RA), Hashimoto's thyroiditis, systemic lupus erythematosus (SLE), and Sjögren's syndrome (SS) [17-20]. In this study, we determined the levels of sGITRL and sGITR in the plasma of MG patients and HDs by ELISA. Our study found that sGITRL showed no difference among all 

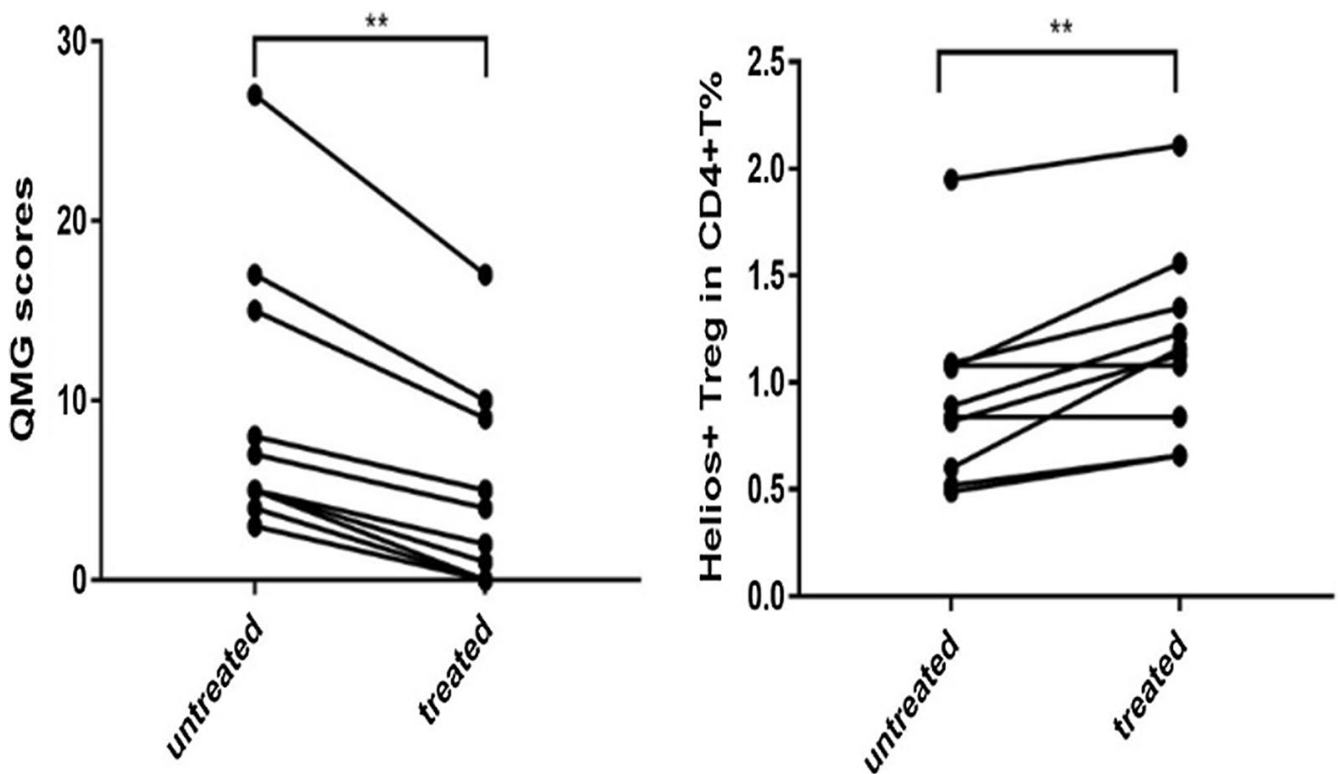

Fig. 2 Change of QMG scores and frequency of Helios ${ }^{+}$Tregs after glucocorticoid therapy in MG patients $(n=10)$. Although the QMG scores significantly decreased after therapy $(p=0.0039)$, frequency of Helios ${ }^{+}$Tregs showed no difference $(p=0.3125) .\left({ }^{*} p<0.05,{ }^{* *} p<0.01,{ }^{* * *} p<0.005\right.$, $\left.{ }^{* * * *} p<0.001\right)$

Table 2 Details of 10 patients and 10 heathy donors

\begin{tabular}{lll}
\hline Groups & MG & HD \\
\hline Age (mean \pm SD) & $32.9 \pm 13.3$ & $36.4 \pm 9.1$ \\
Sex (male/female) & $5 / 5$ & $4 / 6$ \\
QMG score(mean \pm SD) & $10.3 \pm 5.4$ & \\
MGFA I & 3 & \\
MGFA II & 6 & \\
MGFA III & 1 & \\
AchR-Ab positive & 9 & \\
MuSK-Ab positive & 0 & \\
Seronegtive & 1 & \\
Thymoma & 0 & \\
\hline
\end{tabular}

MG myasthenia gravis, HD healthy donors, MGFA Myasthenia Gravis Foundation of America Clinical Classification, QMG score quantitative myasthenia gravis score, AchR-Ab nicotinic acetylcholine receptor antibody, MuSK-Ab muscle specific tyrosine kinase antibody

groups (median values $1201 \mathrm{pg} / \mathrm{mL}$ vs. $1309 \mathrm{pg} / \mathrm{mL}$ vs. $867.9 \mathrm{pg} / \mathrm{mL}, p=0.8250$ ). However, plasma levels of sGITR in GMG were significantly lower than those in HDs (mean $=1522 \pm 115 \mathrm{pg} / \mathrm{mL}$ vs. $2043 \pm 175.4 \mathrm{pg} / \mathrm{mL}$ $p=0.0002$ ) and OMG patients (mean $=1522 \pm 115 \mathrm{pg} /$ $\mathrm{ml} \quad$ vs. $2392 \pm 133.7 \mathrm{pg} / \mathrm{ml}, \quad p=0.0287)$, with no difference between OMG patients and HDs $($ mean $=2392 \pm 133.7 \mathrm{pg} / \mathrm{ml}$ vs. $2043 \pm 175.4 \mathrm{pg} / \mathrm{ml}$, $p=0.10$ ) (Fig. 4). A Spearman correlation analysis was also performed to investigate the relationships between the levels of sGITR and Helios ${ }^{+}$Treg frequency. We found that sGITR positively correlated with Helios ${ }^{+}$ Treg frequency $(\mathrm{r}=0.4001, p=0.0285)$.

\section{GITR directly promoted Helios expression and function in Tregs in vitro}

Our previous data indicated that GITR was associated with frequency of Helios ${ }^{+}$Tregs and severity of MG. This observation suggests that GITRL and GITR modulate Helios expression and further affect suppressive function-related molecules expression in Tregs. To test this hypothesis, we first investigated the direct effect of GITRL and GITR in Tregs. PBMCs from 5 eligible MG patients that received lymphoplasmapheresis therapy were collected to isolate Tregs by magnetic separation. Details of the patients are listed in Table 3, and the purity of $\mathrm{CD}^{+} \mathrm{CD} 25^{+} \mathrm{FOXP3}{ }^{+}$Tregs is demonstrated in Fig. 5e. The purified Tregs were cultured and treated with GITRL $(0.2 \mu \mathrm{g} / \mathrm{mL}, 1 \mu \mathrm{g} / \mathrm{mL}, 5 \mu \mathrm{g} / \mathrm{mL})$ or GITR-Fc $(0.4 \mu \mathrm{g} / \mathrm{mL}$, $2 \mu \mathrm{g} / \mathrm{mL}, 10 \mu \mathrm{g} / \mathrm{mL}$ ) for $72 \mathrm{~h}$. Cells treated with Heatinactivated GITRL $(5 \mu \mathrm{g} / \mathrm{mL})$ or human IgG $(10 \mu \mathrm{g} / \mathrm{mL})$ or none of them were used as controls. Flow cytometry was performed to analyze the expression of Helios. We found that GITRL had no significant effect in promoting Helios expression. However, GITR-Fc promoted Helios expression level $(p<0.0001)$, and this upregulation was in a dose-dependent manner (post linear trend test, $p<0.0001$ ), as shown in Fig. $5 \mathrm{a}-\mathrm{d}$. Then, the expression of FOXP3, CD39, CTLA-4, and PD-L1 in Tregs was 


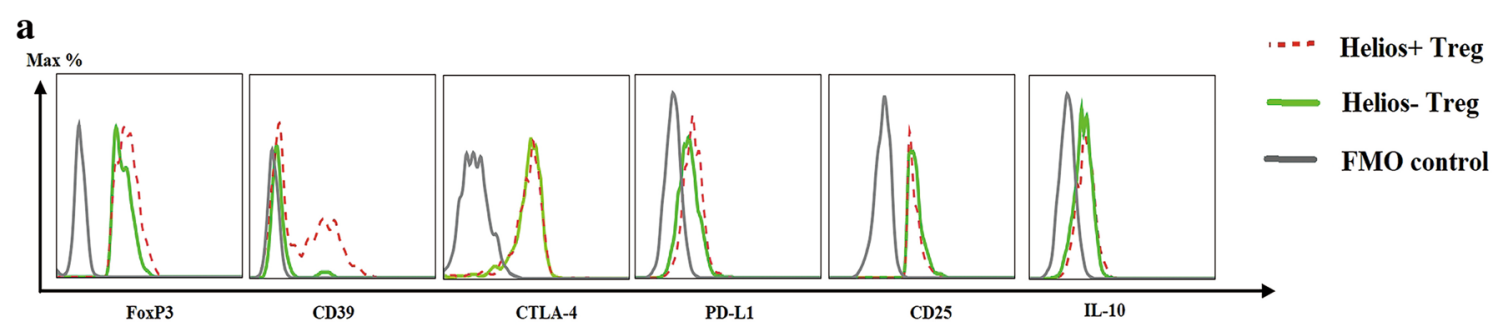

b

MG patients
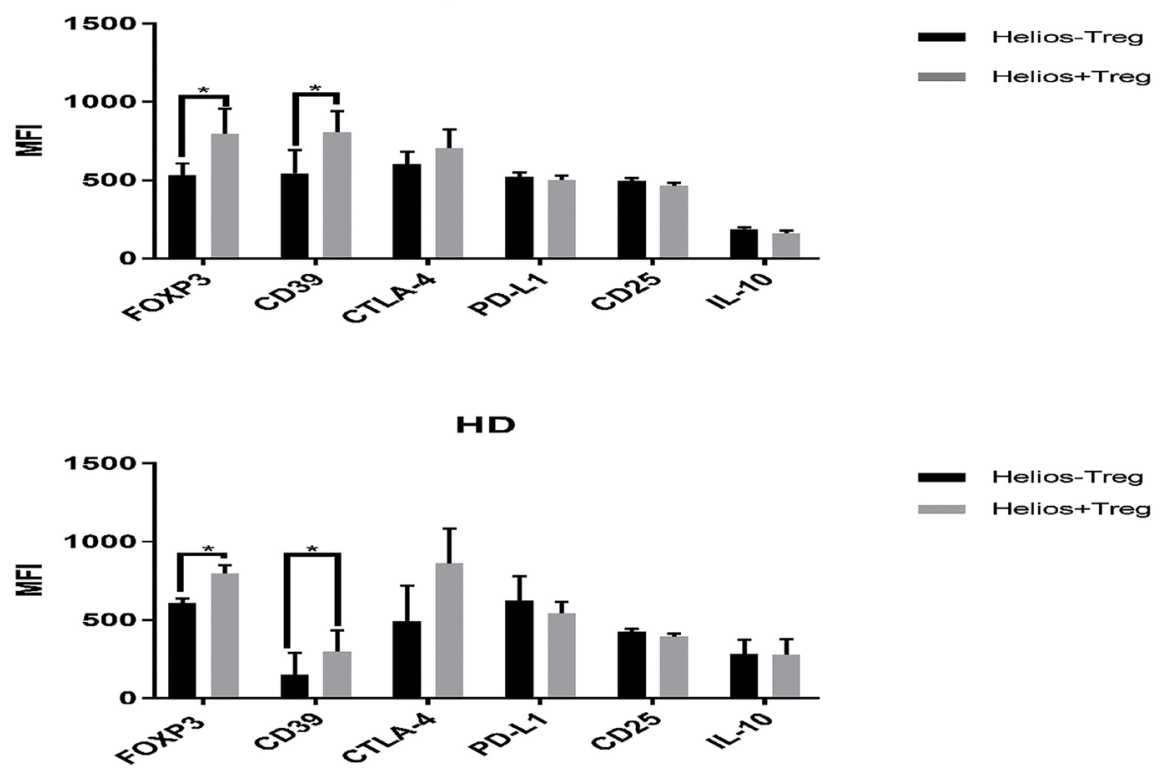

Fig. 3 Analysis of suppressive function-related molecules in $\mathrm{Helios}^{+}{ }^{-}$regs and Helios ${ }^{-}$Tregs. a Histogram showing the representative comparison of FOXP3, CD39, CTLA-4, PD-L1, CD25 and IL-10 expression in Helios ${ }^{+}$Tregs and Helios ${ }^{-}$Tregs. $\mathbf{b}$ Helios $^{+}$Tregs expressed higher levels of FOXP3 and $\operatorname{CD} 39\left(p=0.033, p=0.0415\right.$, respectively) in MG patients and HDs. $\left({ }^{*} p<0.05,{ }^{* *} p<0.01,{ }^{* * *} p<0.005,{ }^{* * *} p<0.001\right)$
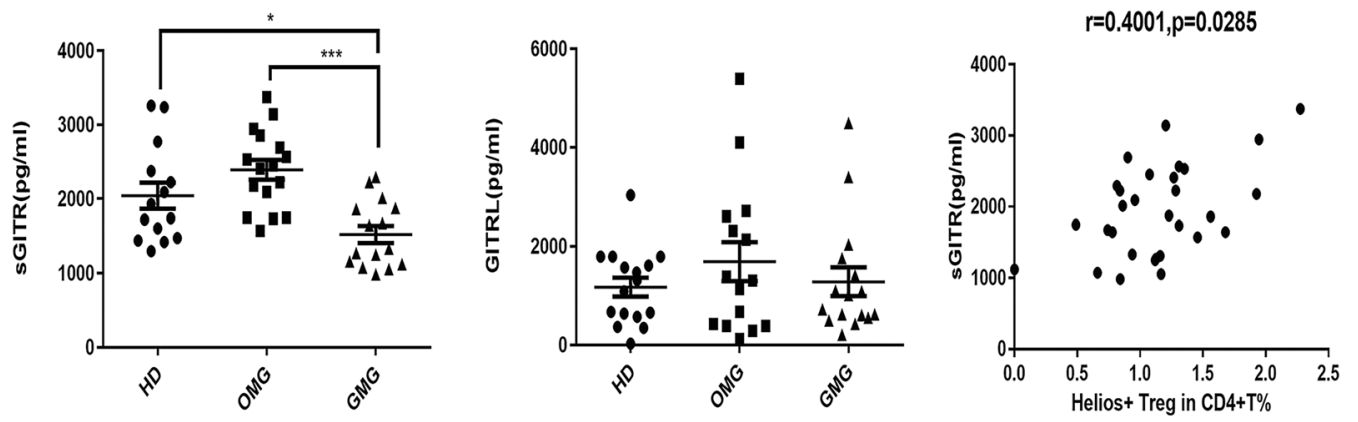

Fig. 4 Level of soluble GITR and soluble GITRL in peripheral plasma. Soluble GITR in GMG patients was significantly lower than in HDs ( $p=0.0377$ ) and OMG patients $(p=0.0002$ ). For soluble GITRL, there was no significant difference among HDs, OMG patients, and GMG patients $(p=0.8250)$. There was a positive correlation between soluble GITR and frequency of Helios ${ }^{+}$regulatory T cells $(r=0.4001, p=0.0285) .{ }^{*} p<0.05$, ${ }^{* *} p<0.01$, $\left.{ }^{* *} p<0.005,{ }^{* * *} p<0.001\right)$ 
Table 3 Details of patients accepted lymphoplasmapheresis

\begin{tabular}{|c|c|c|c|c|c|c|c|}
\hline No. & Age (years) & Gender & QMG score & MGFA classification & Antibody status & Thymoma & MV \\
\hline 1 & 48 & Female & 19 & IV & $A c h R-A b(+)$ & No & No \\
\hline 2 & 32 & Male & 33 & IV & $A c h R-A b(+)$ & No & No \\
\hline 3 & 57 & Male & 32 & IV & AchR-Ab (+) & No & No \\
\hline 4 & 23 & Female & 29 & IV & $A c h R-A b(+)$ & No & No \\
\hline 5 & 47 & Male & 22 & V & MuSK-Ab(+) & No & Yes \\
\hline
\end{tabular}

MGFA Classification Myasthenia Gravis Foundation of America Clinical Classification, QMG score quantitative myasthenia gravis score, AchR-Ab nicotinic acetylcholine receptor antibody, MUSK-Ab muscle specific tyrosine kinase antibody, MV mechanical ventilation

analyzed after treatment with GITR-Fc $(10 \mu \mathrm{g} / \mathrm{mL})$. Both FOXP3 and CTLA-4 expression were increased (Fig. 5f).

\section{GITR regulated Helios expression independent of membrane GITRL in Tregs}

Although we demonstrate that sGITR regulates Helios expression, the underlying mechanism remains unclear. Considering that reverse signaling through membrane GITRL after engagement by sGITR is involved in the inflammatory response in macrophages and dendritic cells [21, 22], we speculated that sGITR may function in Tregs in a similar way. Thus, we investigated GITRL expression on the surface of Tregs. Flow cytometry was used to detect membrane GITRL and FMO, and isotype was used to establish positivity. We found that Tregs, both in MG patients $(n=3)$ and HDs $(n=3)$, did not express membrane GITRL, even when activated by precoating with anti-CD3 mAb, as shown in Fig. 6.

\section{Discussion}

In this study, we show that both frequency of Helios ${ }^{+}$ Tregs and level of sGITR are decreased in GMG patients, as compared to HDs and OMG patients, and are correlated with the severity of MG. Our results also indicate that GITR, not GITRL, promoted expression of Helios and enhanced the modulatory function of Tregs in MG. In addition, we found that GITR may regulate Helios expression independent of membrane GITRL.

We demonstrate an abnormal decrease of $\mathrm{Helios}^{+}$ Tregs in MG. It is known that human Tregs comprise a specific subpopulation of $\mathrm{T}$ cells, which play an essential role in immune homeostasis. Many autoimmune diseases are associated with homeostatic imbalance of regulatory and conventional effector $\mathrm{T}$ cells. However, a unique marker to define Tregs is still lacking. Previously defined markers for the identification of Tregs in MG patients, including CD25, FOXP3, and GITR [3, 4], are also increased during effector $\mathrm{T}$ cell activation; and a potent suppressive subset of Tregs cannot be characterized by these markers. To analyze the contribution of human Tregs to the pathogenesis of MG or any other autoimmune disease, it is necessary to have reliable criteria to accurately determine functional Tregs. Helios, an Ikaros transcription family member, has been recently suggested to be a marker for naturally occurring Tregs. It has become evident that Helios ${ }^{+}$Tregs, in contrast to Helios $^{-}$Tregs, have enhanced suppressive potential [23], migratory potential into inflamed tissues, and possess a highly demethylated Treg-specific demethylated region (TSDR) in the FOXP3 gene, suggesting a more stable phenotype. Helios may therefore define a subset of Tregs with a putative role in mediating self-tolerance. $\mathrm{Xu}$ et al. also found an abnormal decrease of Helios ${ }^{+}$Tregs in MG [4]. However, in other autoimmune diseases, Alexander et al. and Takatori et al. reported that Helios ${ }^{+}$Tregs were expanded in active SLE but unaltered in RA [7, 24], These conflicting reports indicate the lack of knowledge regarding the functionality and the role of Helios-expressing Tregs in autoimmune diseases. The present study investigated Helios expression in Tregs from patients with MG. We found that frequencies of Helios ${ }^{+}$Tregs were significantly decreased in GMG patients compared to HDs and OMG patients, and were negatively correlated with disease severity. Further analysis of markers involved in immunosuppressive activity shows that Helios expressing-Tregs expressed higher level of CD39 and FOXP3. These observations suggest that abnormal reduction of Helios $^{+}$Tregs may contribute to the pathophysiology and development of MG. The comparable frequencies of Helios ${ }^{+}$Tregs between OMG patients and HDs may be due to our limited sample size (14 HDs and 16 OMG patients).

GITRL and GITR are members of the TNF/TNFR superfamily. Their contribution to immune system regulation is complex and important, as demonstrated by experimental models of autoimmunity, inflammation, and tumors. Abnormal elevated levels of GITRL or/and GITR in serum have been reported in SLE, RA, Sjögren's syndrome, and Hashimoto's thyroiditis, which all correlated with disease severity or level of autoantibody [1720]. However, to our knowledge, few studies have focused on the immunological roles of soluble GITRL/GITR involved in MG. In this study, we found levels of GITRL in plasma were comparable in HDs, OMG, and GMG 


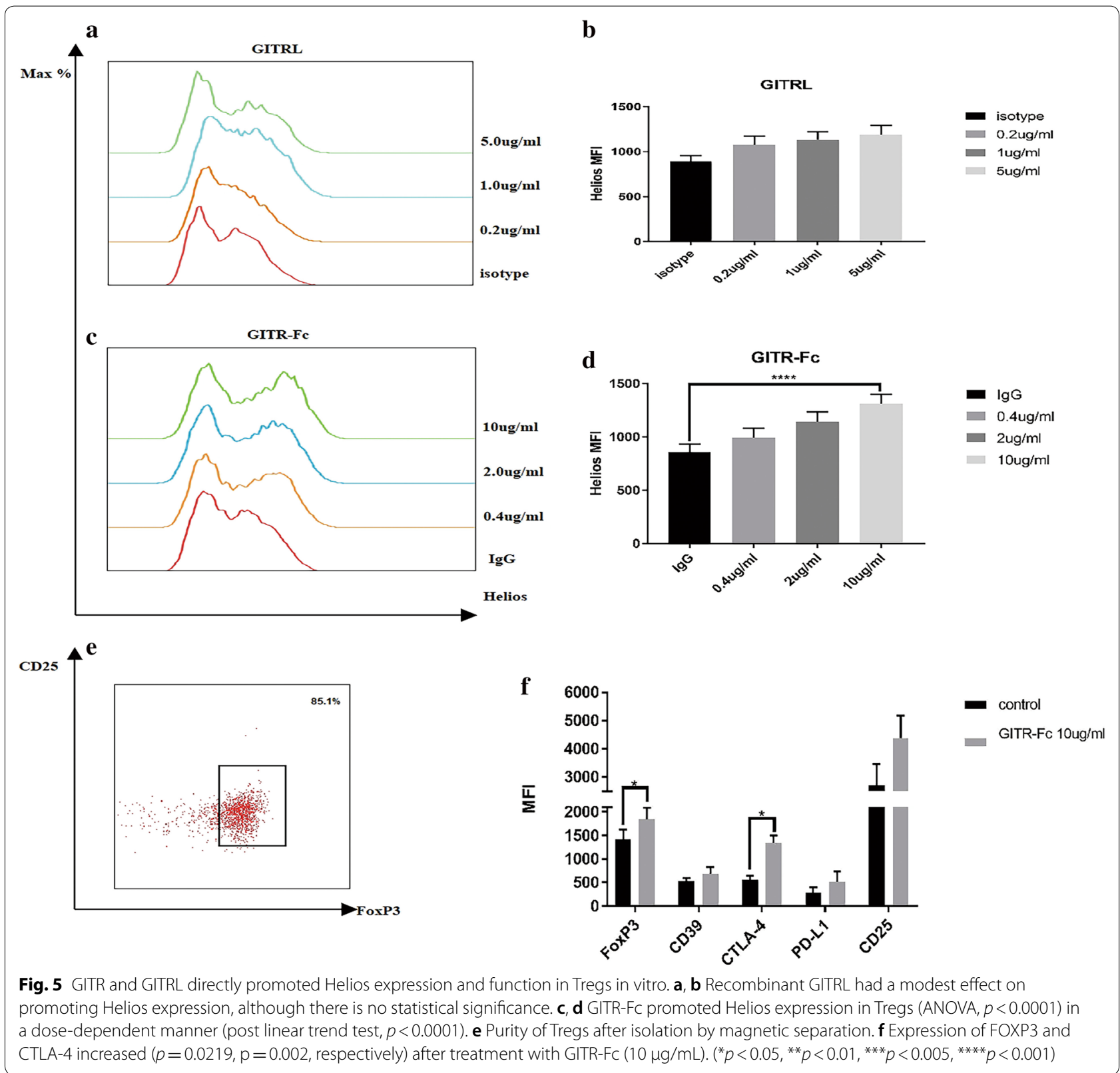

patients while GITR levels in GMG patients were significantly lower than in both HDs and OMG patients, and negatively correlated with QMG scores. Further analysis indicated that GITR was associated with the frequency of Helios ${ }^{+}$Tregs. The association between Helios ${ }^{+}$Tregs and soluble GITR drew our attention to the regulatory effect of GITRL/GITR.

How GITRL and GITR modulate immune reaction remains unclear. Previous research implies that GITRL induces immune response and inhibits activity of Tregs, while Kim et al. showed that GITRL signal is context-dependent, having stimulatory effects on effector $\mathrm{T}$ cells and inhibitory effects through Tregs [15]. Our observation indicates that human GITRL did not impair Tregs function [15]. The modulatory effect of GITR-Fc has been observed previously. Nocentini et al. and Galuppo et al. noticed anti-inflammatory effects of GITR-Fc $[25,26]$; however, the underlying mechanism was unknown. Galuppo et al. demonstrated that GITRFc reduced inflammation similar to the genetic inhibition of GITR expression in mice and they speculated that GITR-Fc exerts its effect depending on the neutralization of GITRL, similar to the effect of anti-mGITRL Ab [12]. However, our results show that GITR-Fc may ameliorate 


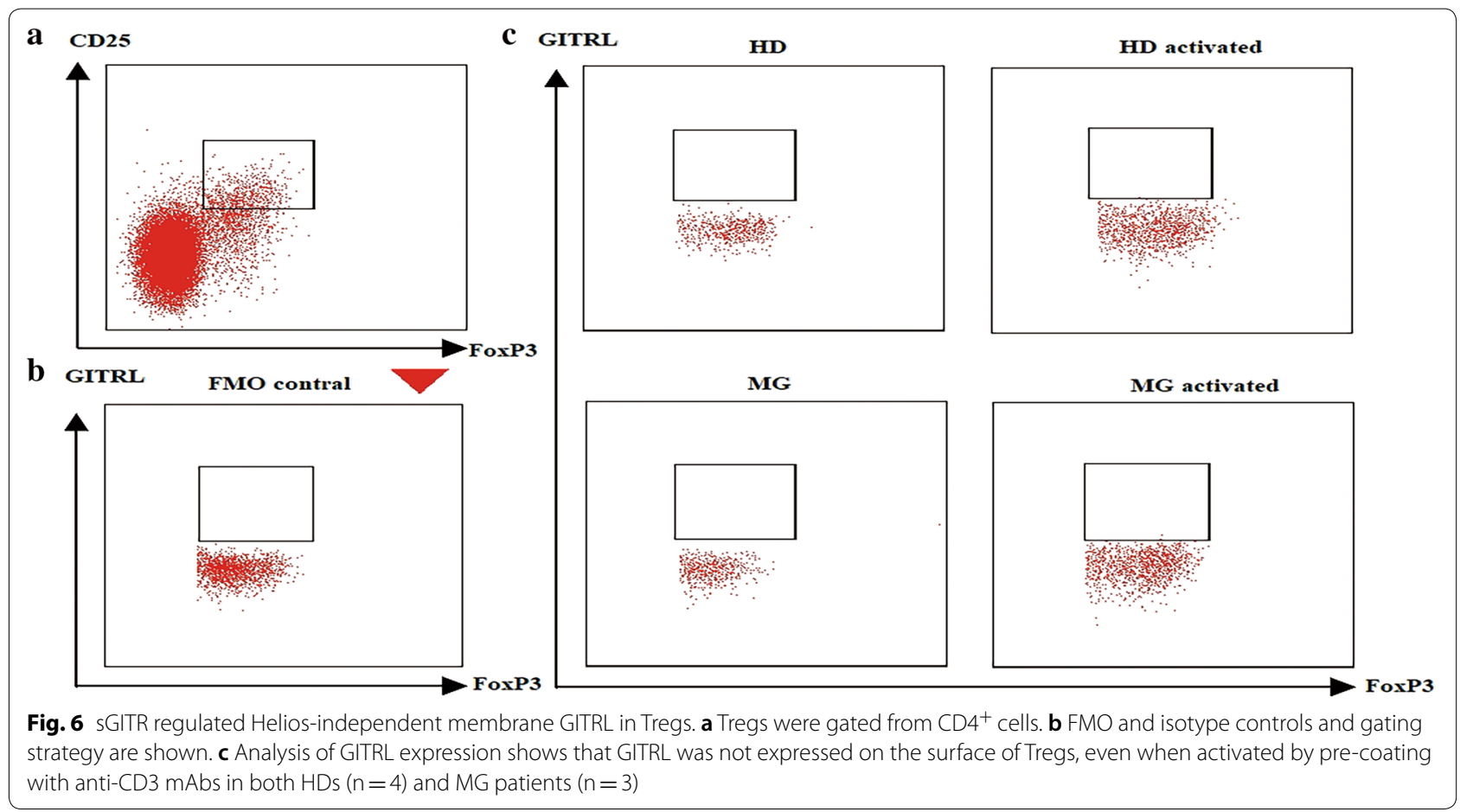

immune response/inflammation by directly enhancing the function of Tregs rather than neutralization of GITRL. We also detected expression of membrane GITRL on Tregs, further indicating that GITR affecting Treg function. In contrast to macrophages or dendritic cells which affected by GITR through membrane GITRL reverse signaling, GITR exert its function independent of membrane GITRL [21, 22]. The signaling pathway of GITR in the context of Tregs remains unknown; however, we speculate that there may exist unknown GITR receptor on Tregs. More studies are required to confirm this hypothesis.

This study has several limitations. First, the limit sample size hindered us to discriminate some small difference, such as whether the frequency of Helios ${ }^{+}$Tregs differed in OMG patients and HDs. Second, we could not directly compare suppressive activity of $\mathrm{Helios}^{+} /$ Helios $^{-}$Tregs through co-culture with effector T cells as Helios is a nuclear transcription factor. Third, the purity of Tregs isolated by MACS was not satisfactory and effect of non-Treg cells cannot be completely excluded. It may be improved by fluorescence activated cell sorting.

\section{Conclusion}

In conclusion, our data show that both frequency of Helios $^{+}$Tregs and level of sGITR are decreased in GMG, compared to HDs and OMG, and correlate with severity of MG. Our data also indicate that GITR directly regulates expression of Helios in MG. This work provides new insight into the regulatory pathway of Helios by GITR and the pathogenesis of MG.

\section{Abbreviations}

MG: myasthenia gravis; Tregs: regulatory T cells; GITR: glucocorticoid-induced tumor necrosis factor receptor; GITRL: glucocorticoid-induced tumor necrosis factor receptor ligand; TNF/TNFR: tumor necrosis factor/tumor necrosis factor receptor; RNS: repetitive nerve stimulation; HD: healthy donors; SGITR/sGITRL: soluble GITR/GITRL; OMG: ocular MG; GMG: generalized MG; MGFA: Myasthenia Gravis Foundation of America; QMG score: quantitative myasthenia gravis score; AchR-Ab: nicotinic acetylcholine receptor antibody; MuSK-Ab: muscle specific tyrosine kinase antibody; PBMCs: peripheral blood mononuclear cells; FMO: fluorescence minus one; PBS: phosphate buffer saline; RA: rheumatoid arthritis; SLE: systemic lupus erythematosus; SS: Sjögren's syndrome; TSDR: Treg specific demethylated region.

\section{Acknowledgements}

This work was also supported by the nurses affiliated to outpatients clinic of department of neurology in Xiangya Hospital.

\section{Authors' contributions}

YL conducted most of the experiments. SY, ZL, HM, and WJ assessed the patients collected the blood samples. HY and WY contributed to the study design and analyzed and interpreted the data. YL drafted the manuscript. HY and WY provided financial support. All authors agree with the publication of the manuscript. All authors read and approved the final manuscript.

\section{Funding}

This study was supported by grants from the National Nature Science Foundation of China (Grant Numbers: 81571173, 81601048 and 8177051973 ).

\section{Availability of data and materials}

The datasets used and/or analyzed during the current study are available from the corresponding author on reasonable request. 


\section{Ethics approval and consent to participate}

All procedures followed were in accordance with the ethical standards of the responsible committee on animal and human experimentation (the Ethics Review Committee of Xiangya Hospital) and with the Helsinki Declaration of 1964 and later versions. Informed consent was obtained from all individual participants.

\section{Consent for publication}

Not applicable.

\section{Competing interests}

The authors declare that they have no competing interests.

Received: 11 October 2018 Accepted: 10 May 2019

Published online: 22 May 2019

\section{References}

1. Gilhus NE, Verschuuren JJ. Myasthenia gravis: subgroup classification and therapeutic strategies. Lancet Neurol. 2015;14:1023-36.

2. Grant CR, Liberal R, Mieli-Vergani G, Vergani D, Longhi MS. Regulatory T-cells in autoimmune diseases: challenges, controversies and-yetunanswered questions. Autoimmun Rev. 2015;14:105-16.

3. Masuda M, Matsumoto M, Tanaka S, Nakajima K, Yamada N, Ido N, et al. Clinical implication of peripheral $\mathrm{CD}^{+}{ }^{+} \mathrm{CD} 25^{+}$regulatory $T$ cells and Th17 cells in myasthenia gravis patients. J Neuroimmunol. 2010;225:123-31.

4. Xu WH, Zhang AM, Ren MS, Zhang XD, Wang F, Xu XC, et al. Changes of Treg-associated molecules on $\mathrm{CD}^{+}{ }^{+} \mathrm{CD} 25^{+}$Treg cells in myasthenia gravis and effects of immunosuppressants. J Clin Immunol. 2012;32:975-83.

5. Shevach EM, Thornton AM. tTregs, pTregs, and iTregs: similarities and differences. Immunol Rev. 2014;259:88-102.

6. Singh K, Hjort M, Thorvaldson L, Sandler S. Concomitant analysis of Helios and Neuropilin-1 as a marker to detect thymic derived regulatory T cells in naïve mice. Sci Rep. 2015;5:7767.

7. Takatori H, Kawashima H, Matsuki A, Meguro K, Tanaka S, Iwamoto T, et al. Helios enhances Treg cell function in cooperation with FoxP3. Arthritis Rheumatol. 2015:67:1491-502.

8. Nakagawa H, Sido JM, Reyes EE, Kiers V, Cantor H, Kim HJ. Instability of Helios-deficient Tregs is associated with conversion to a T-effector phenotype and enhanced antitumor immunity. Proc Natl Acad Sci USA. 2016;113:6248-53.

9. Petrillo MG, Ronchetti S, Ricci E, Alunno A, Gerli R, Nocentini G, et al. GITR + regulatory T cells in the treatment of autoimmune diseases. Autoimmun Rev. 2015:14:117-26.

10. Kim JD, Choi BK, Bae JS, Lee UH, Han IS, Lee HW, et al. Cloning and characterization of GITR ligand. Genes Immun. 2003:4:564-9.

11. Tone M, Tone Y, Adams E, Yates SF, Frewin MR, Cobbold SP, et al. Mouse glucocorticoid-induced tumor necrosis factor receptor ligand is costimulatory for T cells. Proc Natl Acad Sci USA. 2003;100:15059-64.

12. Nocentini G, Ronchetti S, Petrillo MG, Riccardi C. Pharmacological modulation of GITRL/GITR system: therapeutic perspectives. Br J Pharmacol. 2012;165:2089-99.
13. Joetham A, Ohnishi H, Okamoto M, Takeda K, Schedel M, Domenico J, et al. Loss of T regulatory cell suppression following signaling through glucocorticoid-induced tumor necrosis receptor (GITR) is dependent on c-Jun N-terminal kinase activation. J Biol Chem. 2012;287:17100-8.

14. Kim YH, Shin SM, Choi BK, Oh HS, Kim CH, Lee SJ, et al. Authentic GITR signaling fails to induce tumor regression unless Foxp3+ regulatory $T$ cells are depleted. J Immunol. 2015:195:4721-9.

15. Schaer DA, Budhu S, Liu C, Bryson C, Malandro N, Cohen A, et al. GITR pathway activation abrogates tumor immune suppression through loss of regulatory T cell lineage stability. Cancer Immunol Res. 2013;1:320-31.

16. Knee DA, Hewes B, Brogdon JL. Rationale for anti-GITR cancer immunotherapy. Eur J Cancer. 2016:67:1-10.

17. Gan X, Feng X, Gu L, Tan W, Sun X, Lv C, et al. Correlation of increased blood levels of GITR and GITRL with disease severity in patients with primary Sjögren's syndrome. Clin Dev Immunol. 2013;2013:340751.

18. Liu Y, Tang $X$, Tian J, Zhu C, Peng H, Rui K, et al. Th17/Treg cells imbalance and GITRL profile in patients with Hashimoto's thyroiditis. Int J Mol Sci. 2014;15:21674-86

19. Li L, Wen W, Jia R, Li Y, Liu X, Sun X, et al. GITRL is associated with increased autoantibody production in patients with rheumatoid arthritis. Clin Rheumatol. 2016;35:2195-202.

20. Gu L, Xu L, Zhang X, Tan W, Wang H, Zhang M. Correlation of circulating glucocorticoid-induced TNFR-related protein ligand levels with disease activity in patients with systemic lupus erythematosus. Clin Dev Immunol. 2012;2012:265868.

21. Grohmann U, Volpi C, Fallarino F, Bozza S, Bianchi R, Vacca C, et al. Reverse signaling through GITR ligand enables dexamethasone to activate IDO in allergy. Nat Med. 2007;13:579-86.

22. Bae EM, Kim WJ, Suk K, Kang YM, Park JE, Kim WY, et al. Reverse signaling initiated from GITRL induces NF-kappaB activation through ERK in the inflammatory activation of macrophages. Mol Immunol. 2008:45:523-33.

23. Kim HJ, Barnitz RA, Kreslavsky T, Brown FD, Moffett H, Lemieux ME, et al. Stable inhibitory activity of regulatory $T$ cells requires the transcription factor Helios. Science. 2015;350:334-9.

24. Alexander T, Sattler A, Templin L, Kohler S, Groß C, Meisel A, et al. Foxp3+ Helios + regulatory $T$ cells are expanded in active systemic lupus erythematosus. Ann Rheum Dis. 2013;72:1549-58.

25. Nocentini G, Riccardi C. GITR: a modulator of immune response and inflammation. Adv Exp Med Biol. 2009;647:156-73.

26. Galuppo M, Nocentini G, Mazzon E, Ronchetti S, Esposito E, Riccardi $\mathrm{L}$, et al. GITR gene deletion and GITR-FC soluble protein administration inhibit multiple organ failure induced by zymosan. Shock. 2011;36:263-71.

\section{Publisher's Note}

Springer Nature remains neutral with regard to jurisdictional claims in published maps and institutional affiliations.
Ready to submit your research? Choose BMC and benefit from:

- fast, convenient online submission

- thorough peer review by experienced researchers in your field

- rapid publication on acceptance

- support for research data, including large and complex data types

- gold Open Access which fosters wider collaboration and increased citations

- maximum visibility for your research: over 100M website views per year

At BMC, research is always in progress.

Learn more biomedcentral.com/submissions 